Accepted Manuscript

Settling tracer spheroids in vertical turbulent channel flows

Jingran Qiu, Cristian Marchioli, Helge I. Andersson, Lihao Zhao

PII:

DOI:

S0301-9322(19)30218-6

Reference:

https://doi.org/10.1016/j.ijmultiphaseflow.2019.06.01 IJMF 3059

To appear in:

International Journal of Multiphase Flow

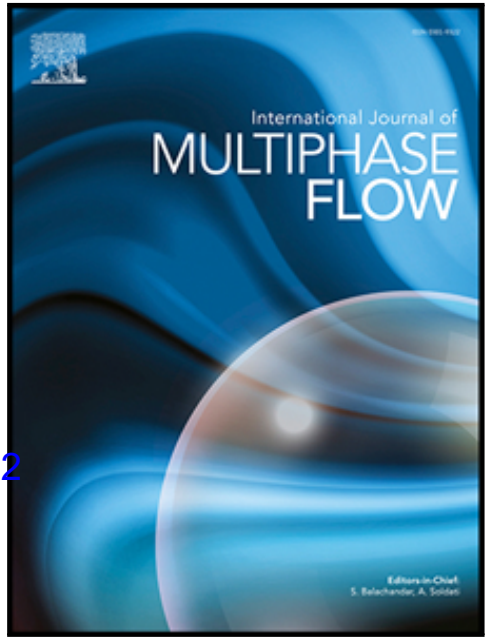

Received date:

5 April 2019

Revised date:

5 June 2019

Accepted date:

24 June 2019

Please cite this article as: Jingran Qiu , Cristian Marchioli , Helge I. Andersson, Lihao Zhao, Settling tracer spheroids in vertical turbulent channel flows, International Journal of Multiphase Flow (2019), doi: https://doi.org/10.1016/j.jjmultiphaseflow.2019.06.012

This is a PDF file of an unedited manuscript that has been accepted for publication. As a service to our customers we are providing this early version of the manuscript. The manuscript will undergo copyediting, typesetting, and review of the resulting proof before it is published in its final form. Please note that during the production process errors may be discovered which could affect the content, and all legal disclaimers that apply to the journal pertain. 


\section{Highlights}

- Enhancement of settling velocity is observed for both oblate and prolate tracer spheroids in downward and upward flow.

- Slip velocity originating from preferential orientation accounts for the major enhancement of settling velocity.

- Particles' preferential sampling of high or low speed flow regions is the result of clustering and influences settling velocity.

- Wall-normal distribution of particle is dominated by wall-normal slip velocity. Transport of particles in the wall-normal direction reaches a stable state when the velocity due to sampling and the slip velocity balance each other. 


\title{
Settling tracer spheroids in vertical turbulent channel flows
}

Jingran Qiu $^{1}$, Cristian Marchioli ${ }^{2,3}$, Helge I. Andersson ${ }^{4}$ and Lihao Zhao ${ }^{1,4}$ a)

${ }^{1}$ AML, Department of Engineering Mechanics, Tsinghua University, Beijing 100084, China

${ }^{2}$ Department of Engineering and Architecture (DPIA), University of Udine, Udine 33100, Italy

${ }^{3}$ Department of Fluid Mechanics, CISM, Udine 33100, Italy

${ }^{4}$ Department of Energy and Process Engineering, Norwegian University of Science and Technology, Trondheim 7491, Norway

a) Electronic mail: zhaolihao@tsinghua.edu.cn

Key words: Spheroidal particles; Wall turbulence; Preferential concentration, Voronoï analysis; Lagrangian point-particle approach

\begin{abstract}
The motion of particles settling in turbulence is an intriguing problem, which is relevant to an indepth understanding of planktons in marine flows or the design of photobioreactors. This work studies the motion, orientation and distribution of inertia-less spheroidal particles settling in vertical channel flows by direct numerical simulations. We show that, compared to spherical tracers, the settling velocity of spheroidal tracers is enhanced due to preferential orientation and local clustering (not due to particle inertia, in the present case). Prolate spheroids tend to align their symmetry axis in the direction of gravity while oblate ones align perpendicular to it. Both kinds of particles attain a larger slip velocity in the direction of gravity and, therefore, settle faster. We also show that particles sample preferentially regions of high fluid velocity in downward flow and regions of low velocity in upward flow. Such preferential sampling, which also contributes to the enhancement of settling, is the result of clustering. Besides, tracer particles are observed to accumulate in the channel center in downward flow and near the wall in upward flow: We show that tracer transport in the wall-normal direction is controlled by the particle-to-fluid slip velocity and by clustering. The slip velocity dominates the transport initially, but tracers increasingly cluster in regions with opposite flow direction as they accumulate either in the channel center or near the wall. Clustering appears to be associate with the coherent structures that characterize wall turbulence, and tracer distribution in the wall-normal direction is found to reach a steady state when the two qualitatively different mechanisms balance each other.
\end{abstract}

\section{INTRODUCTION}

The sedimentation of small particles in turbulent flows is a common but important phenomenon. Particles settling in oceans, such as planktons and organic detritus, are important to the food supply chain of aquatic organisms and for the flux of carbon compounds (Ducklow et al. 1986; Harding 1974; Shanks \& Trent 1980). Other types of particles, in particular anthropogenic micro-plastics are now acknowledged as one of the major sources of environmental pollution (Andrady 2011; Van Cauwenberghe et al. 2013) since they can be ingested by aquatic organisms (Cole et al. 2013) and even accumulate in the human body (Smith et al. 2018). The study of sedimentation and clustering of particles in turbulence might improve current understanding of the dispersion of marine organisms and micro-plastic pollutants. On the other hand, settling and clustering of 
particles are essential processes in the design of photobioreactors, which cultivate phytoplankton and consume solar energy to produce biodiesel (Chen et al. 2011). In particular, clustering might change the exposure of phytoplankton to light, affecting the efficiency of production, whereas settling is an important process in harvesting of biomass (Uduman et al. 2010).

The sedimentation of particles in a quiescent fluid has been studied experimentally (Guazzelli 2006; Herzhaft et al. 1996; Herzhaft \& Guazzelli 1999) and through numerical simulations (Butler \& Shaqfeh 2002; Gustavsson \& Tornberg 2009; Mackaplow \& Shaqfeh 1998). These studies have shown that both prolate and oblate particles exhibit preferential orientation and align with the direction of gravity, forming clusters and streamers in the sedimentation. The mean settling velocity is found to be greater than the theoretical settling velocity in dilute flow conditions but hindered in the semi-dilute regime due to particle-fluid interaction (Herzhaft \& Guazzelli 1999).

However, the motion, distribution and orientation of non-spherical particles are significantly different when they are determined by an underlying turbulent flow (Voth \& Soldati 2017). The particle motion in turbulence has been widely investigated using direct numerical simulations (DNS) of turbulence coupled with the point-particle approach. In the limitation of negligibly small particle Reynolds number, the flow around a particle is Stokesian and the point-particle approach is applicable for particles smaller than Kolmogorov length scale. In spite of the inherent simplifications of the point-particle approach (Cui et al. 2018), interesting physical observations have been made for suspensions of spherical and non-spherical particles in homogeneous isotropic turbulence (Wang \& Maxey 1993; Siewert et al. 2014; Ardekani et al. 2017) and wallbounded turbulence (Zhang et al. 2001; Mortensen et al. 2008; Challabotla et al. 2015a).

The point-particle approach can be applied for both inertial particles and tracer (inertialess) particles. In wall-bounded turbulence, inertial particles are found to accumulate inside the viscous sublayer and cluster in low-speed flow regions (Mortensen et al. 2008; Marchioli et al. 2010). Moreover, the shape of the particles strongly affects their orientation. Inertial prolate particles tend to align their symmetry axes in the velocitygradient plane while oblate particles align in the spanwise direction. In the case of tracer particles with negligible inertia, the particle distribution remains random but preferential orientation is still observed in wallbounded turbulence, namely that the prolate spheroids align in the streamwise direction and oblate ones tend to orient in the wall-normal direction (Challabotla et al. 2015a; Zhao et al. 2015).

The studies in wall-bounded turbulence discussed above do not address the problem of particle sedimentation. The effect of gravity typically results in extra slip velocity and affects particle rotation and/or distribution. Nilsen/et al. (2013), Challabotla et al. (2016) and Yuan et al. (2017) presented direct numerical simulations of vertical channel flows, and suggested that the gravity effect affects particle orientation as well as particle distribution in the wall-normal direction. These studies focused only on particles with moderate inertia, but more recent studies (Ardekani et al. 2017; Fornari et al. 2016; Fornari et al. 2018) have demonstrated that, counter to intuition, gravity has important effects on particles with small inertia. In particular, Ardekani et al. (2017) showed that gravity enhances the settling velocity and preferential clustering of inertia-less particles in down-welling regions of isotropic turbulence.

In this work, we build on previous studies to improve our understanding of gravity effects on nonspherical tracers in wall-bounded turbulence. Previous study (Challabotla et al. 2015a) have shown that such tracers are characterized by a random spatial distribution in wall-bounded turbulence. However, tracers exhibit 
a non-zero gravity-induced velocity relative to the surrounding fluid, which makes it possible to form clusters and further exert influence on other statistics. Therefore, we performed direct numerical simulations of vertical turbulent channel flow to examine the effect of gravity on velocity and orientation of both oblate and prolate tracers, and, more importantly, to reveal the casual relation between these observables.

Simulations are performed using a standard Eulerian-Lagrangian method based on point-particle tracking, and considering three configurations, i.e. downward, upward flow and no-gravity flow. The settling velocity and orientation of large swarms of inertia-less spheroids are examined statistically, and Voronoï analysis (Monchaux et al. 2010) is employed to investigate clustering. In Section 2, the fluid and particle governing equations are introduced. In Section 3, the statistical characterization of settling velocity, orientation and clustering are discussed and put in causal relation. Finally, the main findings are summarized and conclusions are drawn in Section 4.

\section{METHODOLOGY}

\section{A. Eulerian fluid dynamics}

In the present work, two flow configurations with gravity are considered, as shown in figure 1 . In the case of downward flow, the flow direction is the same as gravity direction, while the flow is opposite to gravity in upward flow. A configuration with no gravity is also presented for comparison. Direct numerical simulations are performed by solving the Navier-Stokes equations for isothermal flow of an incompressible Newtonian fluid. In vector form, these equations read as

$$
\begin{aligned}
& \nabla \cdot \mathbf{u}=0, \\
& \frac{\partial \mathbf{u}}{\partial t}+\mathbf{u} \cdot \nabla \mathbf{u}=-\frac{\nabla p}{\rho_{f}}+\nu \nabla^{2} \mathbf{u}-\frac{\mathrm{d} P}{\mathrm{~d} x} \mathbf{e}_{x}+\rho_{f} g \mathbf{e}_{g},
\end{aligned}
$$

where $\mathbf{u}$ is the velocity of fluid. $\rho_{f}, p$ and $y$ denote the fluid density, the dynamic pressure and fluid kinematic viscosity, respectively. The third term on the right of equation 2 represents the effect of mean pressure gradient, where $P$ and $\mathbf{e}_{x}$ denote the mean pressure and unit vector along the steamwise $(x)$ direction. The last term on the right is the gravity term, where $g$ is gravitational acceleration and $\mathbf{e}_{\mathrm{g}}$ denotes the unit vector along the gravity direction. Specifically, $\mathbf{e}_{\mathrm{g}}$ equals to $\mathbf{e}_{\mathrm{x}}$ and $-\mathbf{e}_{\mathrm{x}}$ in downward and upward flow, respectively. The flow is characterized by friction Reynolds number $R e_{\tau}=h u_{\tau} / v$, where $u_{\tau}$ is the wall friction velocity and $h$ is half of the channel height. The sum of the last two terms of equation 2 is the same in all three configurations to keep the flows with the same $R e_{\tau}$.

Simulations for all three configurations are performed at $R e_{\tau}=180$ in a domain of size $12 h \times 6 h \times 2 h$ with $192 \times 192 \times 192$ mesh points in the streamwise $(x)$, span-wise $(y)$, and wall-normal $(z)$ directions, respectively. The mesh resolution is uniform in the streamwise and span-wise directions, with grid spacings $\Delta x^{+}=11.3$ and $\Delta y^{+}=5.6$. In the wall-normal direction, the grid spacing $\Delta z^{+}$ranges from 0.9 (at the channel walls) to 2.86 (in the channel center). The time step is $\Delta t^{+}=0.036$. Periodic boundary conditions are adopted in the homogeneous directions and the no-slip and impermeability conditions are imposed at the walls. The superscript "+" denotes the dimensionless quantity normalized by viscosity length scale $v / u_{\tau}$ or viscosity time scale $v / u_{\tau}^{2}$. A pseudo-spectral method is applied along the homogenous directions and a second-order 
finite-difference discretization is used in the wall-normal direction. Second-order explicit Adams-Bashforth scheme is adopted for the time advancement. The set-up of Eulerian numerical method for fluid phase is the same as those used by Challabotla et al. (2016) and Yuan et al. (2017).

\section{B. Lagrangian particle dynamics}

The asphericity of spheroidal particles is characterized by their aspect ratio $\lambda=c / a$, where $2 c$ is the length of the symmetry axis and $2 a=2 b$ is the length of the two other axes. The characteristic length of particle is smaller than the Kolmogorov length scale and the density of particles is nearly equal to that of the fluid. Therefore, the point particle model adopted in present study can be justified. The particle Stokes number is defined by $\mathrm{St}=\tau_{\mathrm{p}} / \tau_{\mathrm{f}}$, where $\tau_{\mathrm{p}}$ is the translational particle response time and $\tau_{\mathrm{f}}=v / \mathrm{u}_{\tau}{ }^{2}$ is viscous fluid time scale. The specific definition of $\tau_{\mathrm{p}}$ reads (Shapiro \& Goldenberg 1993; Challabotla et al. 2015b)

$$
\tau_{p}=\left\{\begin{array}{cl}
\frac{2 D a^{2}}{9 v} \frac{\lambda\left\{\pi-2 \tan ^{-1}\left[\lambda\left(1-\lambda^{2}\right)^{-1 / 2}\right]\right\}}{2 \lambda\left(1-\lambda^{2}\right)^{1 / 2}} & \lambda \leq 1, \\
\frac{2 D a^{2} \frac{\lambda \ln \left[\lambda+\sqrt{\lambda^{2}-1}\right]}{9 v}}{\sqrt{\lambda^{2}-1}} & \lambda \geq 1 .
\end{array}\right.
$$

The rotational particle time scale is known to be smaller than the translational time scale (Marchioli et al. 2016). As shown in table 1, the particle Stokes number considered in this study is much smaller than unity: This implies that the hydrodynamic Stokes force and Jeffery torques acting on the particle can be safely neglected, thus justifying the tracer particle model adopted here. The equations governing particle translation (Ardekani et al. 2017) read as,

$$
\begin{aligned}
& \frac{\mathrm{d} \mathbf{x}}{\mathrm{d} t}=\left.\mathbf{u}\right|_{p}+\mathbf{v}_{s}, \\
& \mathbf{v}_{s}=v_{\text {settle }}\left(\frac{\pi}{2}\right) \mathbf{e}_{g}+\left[v_{\text {settle }}(0)-v_{\text {settle }}\left(\frac{\pi}{2}\right)\right]\left(\mathbf{e}_{g} \cdot \mathbf{p}\right) \mathbf{p},
\end{aligned}
$$

where $\mathbf{x},\left.\mathbf{u}\right|_{p}, \mathbf{v}_{\mathrm{s}}$ and $\mathbf{p}$ are the particle position, local fluid velocity, slip velocity and orientation vector, respectively. The unit yector $\mathbf{e}_{\mathrm{g}}$ represents the direction of gravity. The vertical component of settling velocity vector in still fluid is denoted as $v_{\text {settle }}$ and depends on the particle orientation relative to the gravity direction (Siewert et al. 2014):

$$
\mathrm{v}_{\text {settle }}\left(\theta_{x}\right)=6 \pi a \lambda\left(\frac{1}{\left(k_{\hat{z} \hat{z}}-k_{\hat{x} \hat{x}}\right) \cos ^{2} \theta_{x}+k_{\hat{x} \hat{x}}}\right) \frac{2 D a^{2} g}{9 v},
$$

with $D=\left(\rho_{p}-\rho_{f}\right) / \rho_{f}$, where $\rho_{p}$ and $\rho_{f}$ are the density of particle and fluid, respectively. $k_{\hat{x} \hat{x}}$ and $k_{\hat{z} \hat{z}}$ are the resistance coefficients (Siewert et al. 2014). According to equation 5, the slip velocity is dependent on the instantaneous particle orientation $\mathbf{p}$. The streamwise slip velocity reaches a maximum value when the orientation $\mathbf{p}$ is orthogonal to the gravity direction $\mathbf{e}_{\mathbf{g}}$ for an oblate particle or when $\mathbf{p}$ is parallel to $\mathbf{e}_{\mathrm{g}}$ for a prolate particle.

The orientation $\mathbf{p}$ is determined (Jeffery 1922) by

$$
\frac{\mathrm{d} \mathbf{p}}{\mathrm{d} t}=\mathbf{\Omega} \cdot \mathbf{p}+\frac{\lambda^{2}-1}{\lambda^{2}+1}[\mathbf{I}-\mathbf{p p}] \cdot \mathbf{S} \cdot \mathbf{p}
$$


where $\boldsymbol{\Omega}$ and $\mathbf{S}$ are the antisymmetric and symmetric parts of the fluid velocity gradient tensor. Periodic boundary conditions for particles are applied in the homogenous directions. Particle-wall collision is fully elastic: After a collision with the wall, the particle keeps its linear momentum in the two homogenous directions and its angular momentum (Marchioli et al. 2010; Mortensen et al. 2008). Collisions between particles are neglected because the suspension is dilute. Both translational and rotational motions are expressed with respect to an inertial frame of reference $x_{i}=(x, y, z)$. We considered spheroids with $\lambda=0.02,0.1$, $0.33,0.67,1.001,3,10$, and 50 . The density ratio $D$ equals to 1.05 and the radii of particles, $a$, are chosen such that the isotropic settling velocity remains constant as we vary the aspect ratio. The isotropic settling velocity, denoted as $v_{\mathrm{s}}^{\text {iso }}$, is defined as the theoretical settling velocity that can be obtained by averaging over an isotropic distribution of orientations in still fluid. The isotropic velocity is thus expressed as

$$
v_{s}^{i s o}=\int_{0}^{2 \pi} \int_{0}^{\pi} \frac{1}{4 \pi} \mathbf{v}_{s} \cdot \mathbf{e}_{g} \sin \theta_{x} d \theta_{x} d \phi,
$$

where $\theta_{x}$ is the angle between the symmetry axis of a particle and the gravity direction, and $\phi$ is the angle between the $y$-axis and the projection of the symmetry axis on the $y$ - $z$ plane. Replacing $\mathbf{v}_{\mathrm{s}}$ with equation 5 yields

$$
v_{s}^{i s o}=\frac{1}{3}\left(v_{\text {settle }}(0)+2 v_{\text {settle }}\left(\frac{\pi}{2}\right)\right) .
$$

(a) Downward flow

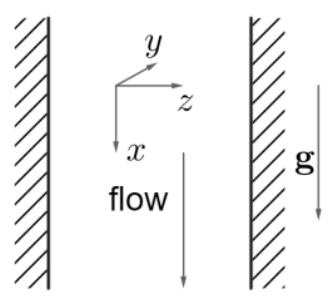

(b) Upward flow

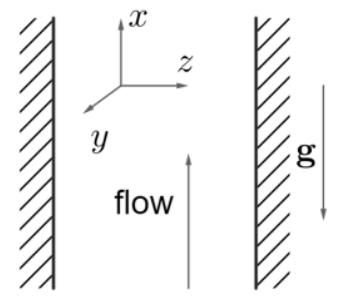

Figure 1. Sketch of flow configurations. (a) Downward flow. (b) Upward flow.

Table 1 Parameters of particles. $v_{s}^{\text {iso }} / u_{\tau}=0.674, \mathrm{St}=0.068$.

\begin{tabular}{ccc}
\hline$\lambda$ & $(\lambda-1) /(\lambda+1)$ & $a^{+}$ \\
\hline 0.02 & -0.961 & 3.074 \\
0.1 & -0.818 & 1.409 \\
0.33 & -0.504 & 0.824 \\
0.67 & -0.198 & 0.623 \\
1.001 & 0.000 & 0.542 \\
1.5 & 0.200 & 0.477 \\
3 & 0.500 & 0.396 \\
10 & 0.818 & 0.312 \\
50 & 0.961 & 0.252 \\
\hline
\end{tabular}

The expression adopted for the isotropic settling velocity is consistent with that of Ardekani et al. (2017), but has been extended to the case of both prolate and oblate particles. Swarms of $N=2 \times 10^{5}$ spheroids are 
randomly injected into a fully-developed turbulent channel flow at $\mathrm{t}^{+}=0$ and the statistics are averaged over a time window from $t^{+}=900$ to $t^{+}=6600$, where $t^{+}=t u_{\tau}^{2} / v$ is the dimensionless time.

\section{RESULTS AND DISCUSSION}

In the present study, we exploit a statistical analysis of particle behavior to examine particle clustering and settling. Non-settling tracer particles have been observed to attain a random distribution in wall normal direction (Challabotla et al. 2015a). However, inhomogeneous distribution is found for particles settling in the vertical flow because of the slip velocity (see equation 4). Figure 2 shows the particle number density for all sets reported in table 1 and indicates that particles tend to accumulate in the center of channel in downward flow (panel a) but accumulate near the wall in upward flow (panel b). These trends are not fully consistent with those observed for inertial particles, which always cluster in the wall region regardless of gravity direction (Challabotla et al. 2016; Marchioli et al. 2010; Nilsen et al. 2013). We also observe that the degree of accumulation increases with particle asphericity, in agreement with previous findings for inertial particles in turbulent channel flow (Challabotla et al. 2016; Marchioli et al. 2010). The accumulation phenomenon shown in figure 2 is observed to occur at the scale of the whole channel. We find that the accumulation is associated with the wall-normal transport of the particles, which results from the presence of non-zero slip velocity and the preferential sampling of specific flow regions by the particles. A detailed discussion will be presented later in this section.
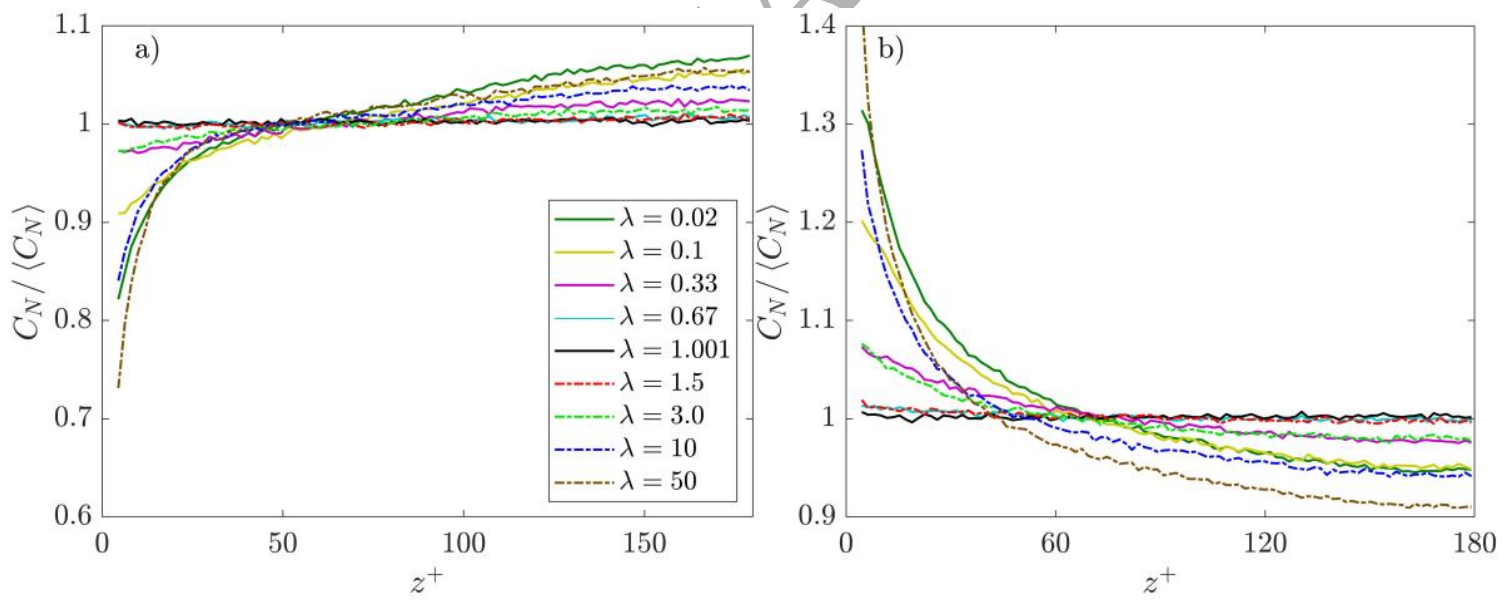

Figure 2. Particle/number density $C_{N}$, normalized by random particle distribution $\left\langle C_{N}\right\rangle$ as a function of wall-normal location $\mathrm{z}^{+}$with different aspect ratios. (a) Downward flow. (b) Upward flow. 

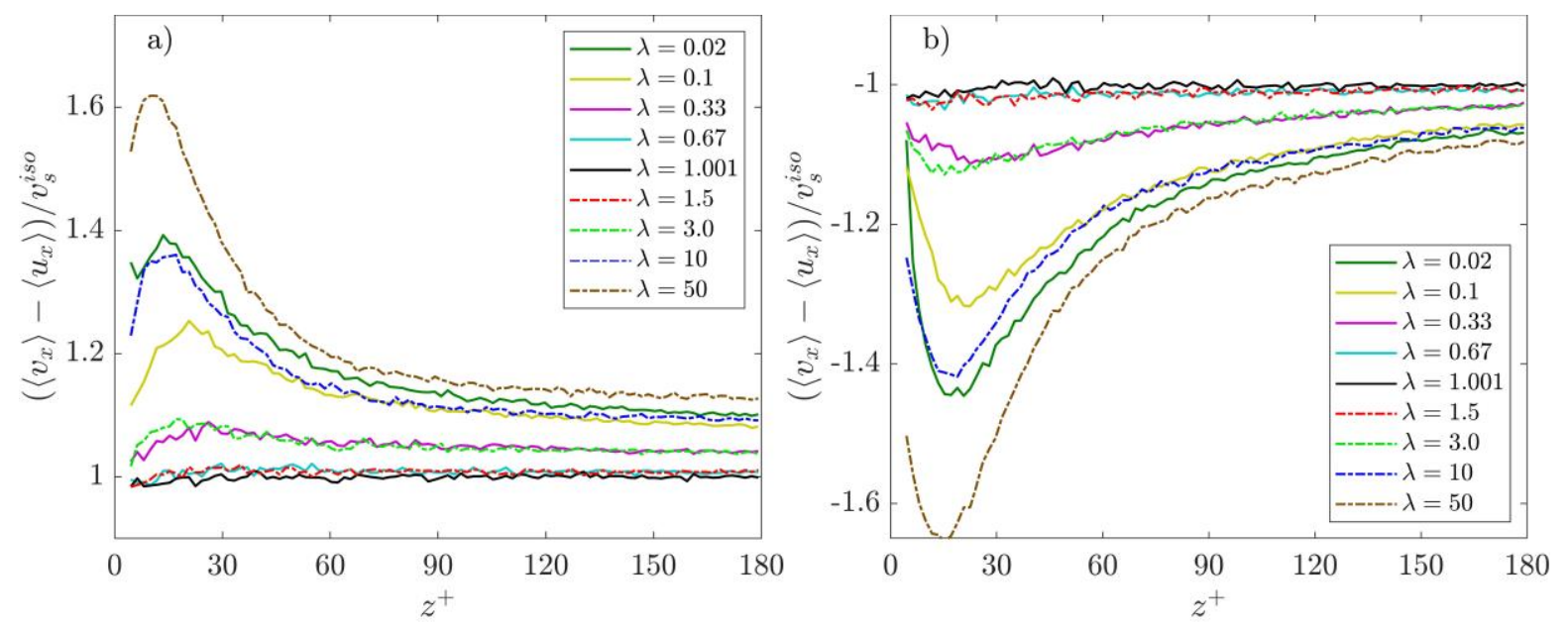

Figure 3. The streamwise settling velocity of particles as a function of wall-normal location $\mathrm{z}^{+}$with different aspect ratios. (a) Downward flow. (b) Upward flow.
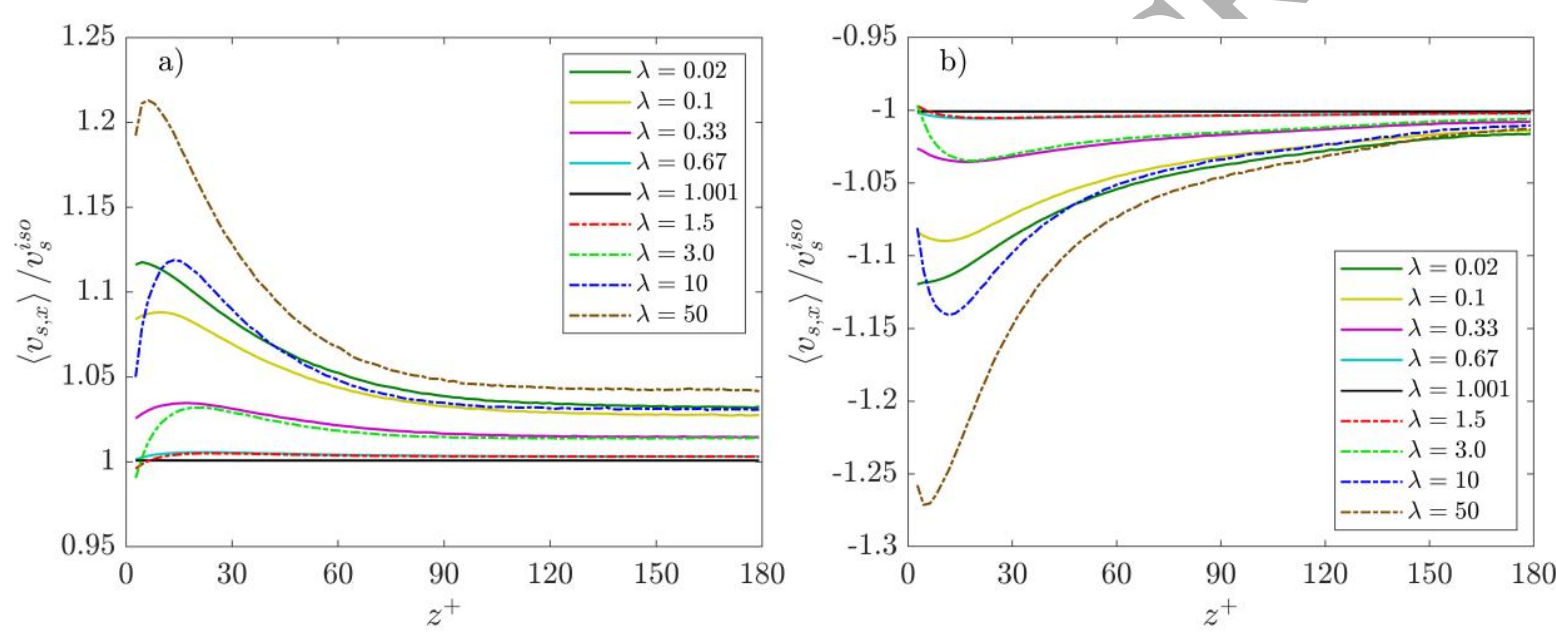

Figure 4. Mean streamwise slip velocity $y_{s, x}$ as function of wall-normal location $\mathrm{z}^{+}$with different aspect ratios. (a) Downward flow. (b) Upward flow. 

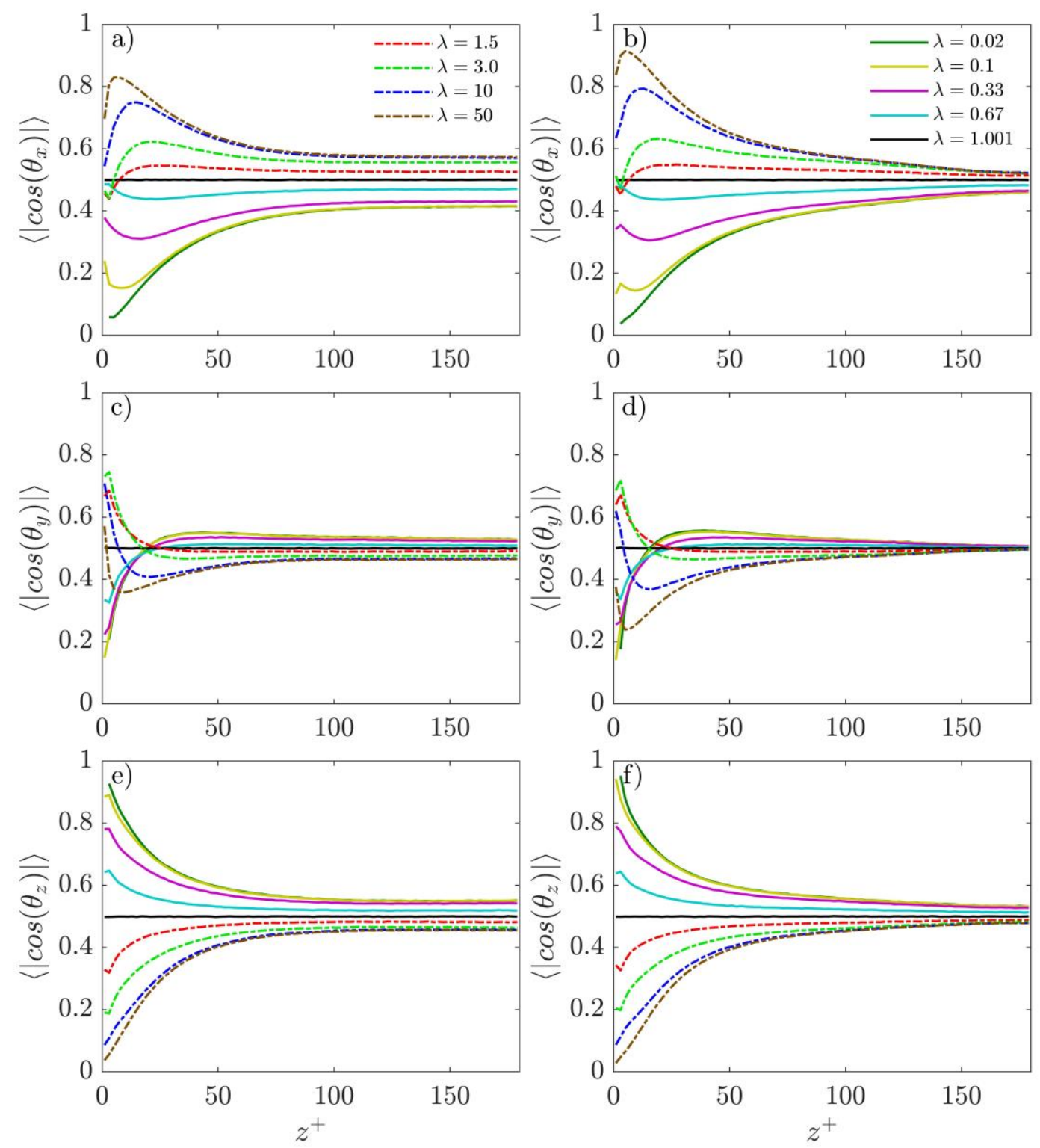

Figure 5. The mean absolute direction cosine of the streamwise (a, b), span-wise (c, d) and wall-normal direction (e, f) as function of wall-normal location $z^{+}$. (a, c, e) Downward flow. (b, d, f) Upward flow.

The settling of particles is examined by considering first their (mean) settling velocity, defined as the difference between the mean particle velocity and the mean Eulerian fluid velocity. In the present work, we use the isotropic settling velocity (given in equation 9) to normalize the settling velocity. The streamwise settling velocity is shown in figure 3. Particles lead the fluid in downward flow but lag behind the fluid in upward flow in the whole channel. As could be expected, spherical particles settle with isotropic settling velocity, but spheroidal particles settle faster. The enhancement of settling velocity, though limited compared to the mean flow velocity, increases with asphericity for both prolate and oblate spheroids, especially in the wall region. The enhanced settling velocity is explained by the two mechanisms of preferential alignment with gravity and the clustering in specific regions of the flow (Ardekani et al. 2017; Herzhaft \& Guazzelli 1999; Mackaplow \& Shaqfeh 1998). The enhancement observed in figure 3 is stronger than the one reported for isotropic turbulence (Ardekani et al. 2017), because preferential alignment diminishes for randomly-oriented particles in isotropic turbulence whereas both mechanisms contribute to the settling of particles in the present 
vertical flow configurations. To examine the relative importance of preferential alignment and clustering, we split the settling velocity into two terms:

$$
\langle\mathbf{v}\rangle-\langle\mathbf{u}\rangle=\left.\Delta \mathbf{U}\right|_{p}+\left\langle\mathbf{v}_{s}\right\rangle
$$

where $\left.\Delta \mathbf{U}\right|_{p}=\left\langle\left.\mathbf{u}\right|_{p}\right\rangle-\langle\mathbf{u}\rangle$.

On the left-hand side of equation 10 is the mean settling velocity, defined as the difference between the mean particle velocity, $\langle\mathbf{v}\rangle$, and the mean Eulerian fluid velocity, $\langle\mathbf{u}\rangle$, computed upon averaging over all grid points at different $z^{+}$. The first term on the right-hand side of equation $10,\left.\Delta \mathbf{U}\right|_{p}$, is the difference between the mean fluid velocity in the Lagrangian frame, $\left\langle\left.\mathbf{u}\right|_{\mathrm{p}}\right\rangle$, (averaged at particle locations) and the Eulerian velocity $\langle\mathbf{u}\rangle$, and so called velocity difference. This term is the result of the particle preferential sampling of high speed or low speed regions. The second term on the right-hand side, $\left\langle\mathbf{v}_{\mathrm{s}}\right\rangle$, is the mean slip velocity (see equation 5), which is determined by the direction of the particle's symmetry axis and thus reflects the preferential orientation. In the following, we discuss $\left\langle\mathbf{v}_{\mathrm{s}}\right\rangle$ and $\left.\Delta \mathbf{U}\right|_{p}$ to analyze the contribution of preferential orientation and local clustering separately.

\section{A. Particle-to-fluid slip velocity}

We first consider the contribution of slip velocity. As shown in figure 4, the slip velocity is larger than or equal to the isotropic settling velocity in the whole channel, and appears to account for a major proportion of the settling velocity. According to equation 5, the streamwise component of slip velocity is determined by the angle between the symmetry axis of the particle and the direction of gravity. As introduced in Methodology, an oblate particle settles fastest when aligning symmetry axis orthogonal to the gravity direction, and a prolate particle settles fastest when aligning symmetry axis parallel to the gravity direction. Therefore, the profiles shown in figure 4 indicate clearly preferential orientation of both prolate and oblate particles. This is confirmed by the behavior of the averaged absolute direction cosines of the particle symmetry axis, shown in figure 5. Regardless of the flow direction, prolate particles (dashed lines) tend to align their symmetry axes with the streamwise direction, while the symmetry axes of oblate particles (solid lines) tend to be orthogonal to the streamwise direction, especially, near the wall. These results are similar to those reported by Challabotla et al. (2015a) for turbulent channel flow without gravity and explain the enhancement of slip velocity compared to isotropic settling velocity.

To highlight the effect of particle shape on the observables just discussed, in figure 6 we show the mean streamwise slip velocity $v_{s, x}$ and the direction cosine $\cos ^{2} \theta_{\mathrm{x}}\left(\cos ^{2} \theta_{\mathrm{y}}\right.$ and $\cos ^{2} \theta_{\mathrm{z}}$ not shown for sake of brevity) versus the shape factor $(\lambda-1) /(\lambda+1)$, which is defined only to increase the legibility of figures in present discussion. In the near-wall region (figures $6 \mathrm{a}, \mathrm{c}$ ), both oblate and prolate particles (with large and small values of the shape factor, respectively) show strong preferential orientation and enhanced slip velocity. The orientation of spheroidal tracers has been explained in terms of Lagrangian fluid stretching (Zhao \& Andersson 2016): Prolate particles (resp. oblate particles) tend to align with the maximum (resp. minimum) eigenvector of the left Cauchy-Green strain tensor, and the alignment increases with asphericity. In the present simulations, the slip velocity does not change this tendency and the orientation is almost unrelated to the gravity direction. Indeed, oblate particles have the same orientation in downward, upward and no-gravity flow, 
and prolate particles show little difference among the three cases as well.

Non-spherical particles also show some preferential orientation in the core region, which is much weaker than near the wall (see figures $6 \mathrm{~b}$, d). Particles in downward flow exhibit stronger preferential orientation than in upward flow and no-gravity flow, which is consistent with the findings of Challabotla et. al. (2016) for inertial particles. Moreover, the preferential orientation increases with asphericity but saturates when $\lambda$ is greater than 10 or less than 0.1 , in agreement with the observations made for isotropic turbulence (Ardekani et al. 2017). This happens because, when particle asphericity increases, the contributions of the fluid strain rate (equation 7) and the anisotropy of settling velocity increase, and both saturate when aspect ratio is sufficiently large or small (Ardekani et al. 2017).

\section{B. Mean velocity difference}

The mean velocity difference $\left.\Delta \mathbf{U}\right|_{p}$ (equation 11) also contributes to the enhancement of the settling velocity. The mean velocity difference is non-zero because spheroidal tracers are allowed to move relative to the fluid as they settle and thus cluster in preferred flow regions. In particular, $\left.\Delta \mathbf{U}\right|_{p}$ is greater (resp. less) than zero when particles sample high-speed (resp. low-speed) regions of the flow, in which the fluid has higherthan-mean (resp. lower-than-mean) velocity at particle position.
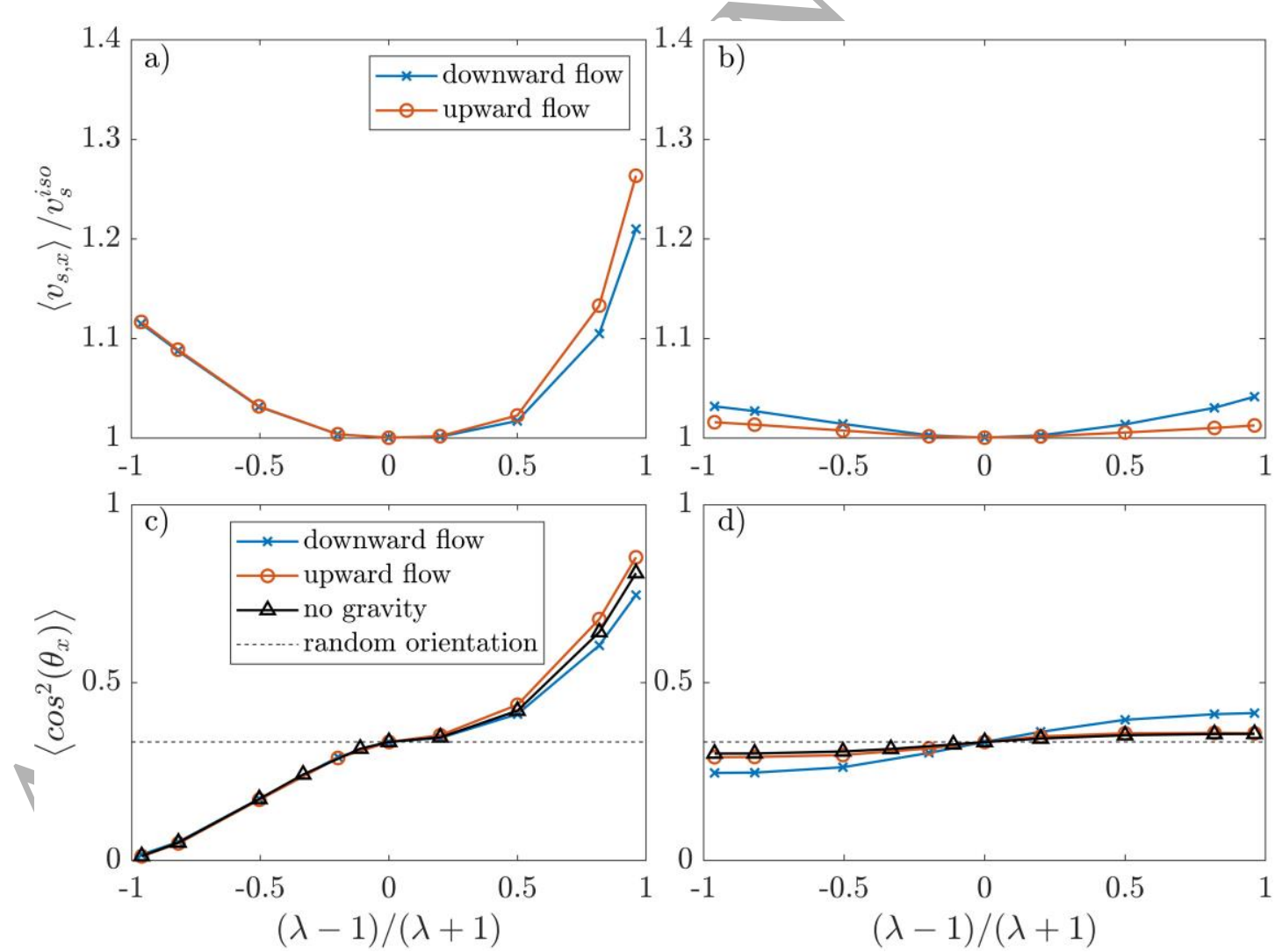

Figure 6. The mean streamwise slip velocity $\left\langle v_{s, x}\right\rangle(\mathrm{a}, \mathrm{b})$ and the direction cosine $\left\langle\cos ^{2}\left(\theta_{x}\right)\right\rangle(\mathrm{c}, \mathrm{d})$ versus the shape factor $(\lambda-1) /(\lambda+1)$. $(\mathrm{a}, \mathrm{c}) \mathrm{z}^{+}=6.3 \sim 9.9$ and $(\mathrm{b}, \mathrm{d}) \mathrm{z}^{+}=176.4 \sim 183.6$. 

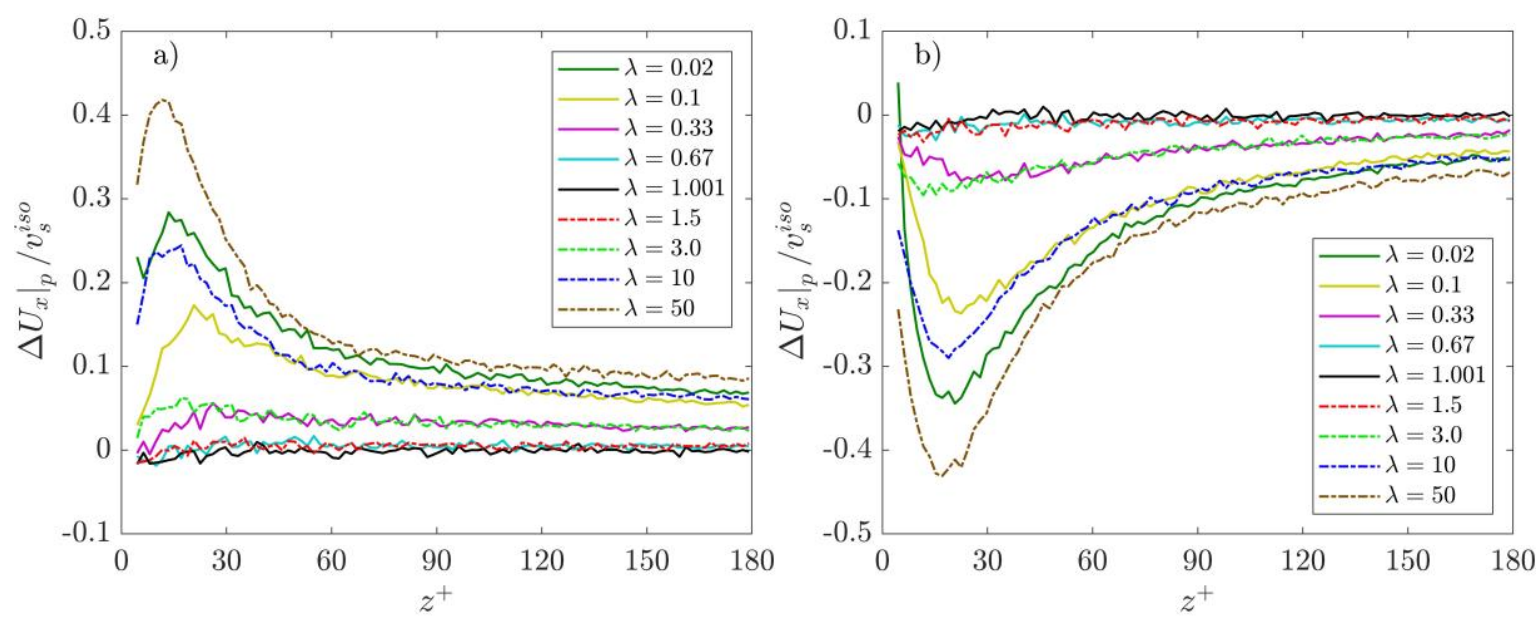

Figure 7. The streamwise fluid velocity difference $\left.\Delta U_{\mathrm{x}}\right|_{p}$ as a function of the wall-normal coordinate $z^{+}$ for different aspect ratios. (a) Downward flow. (b) Upward flow.
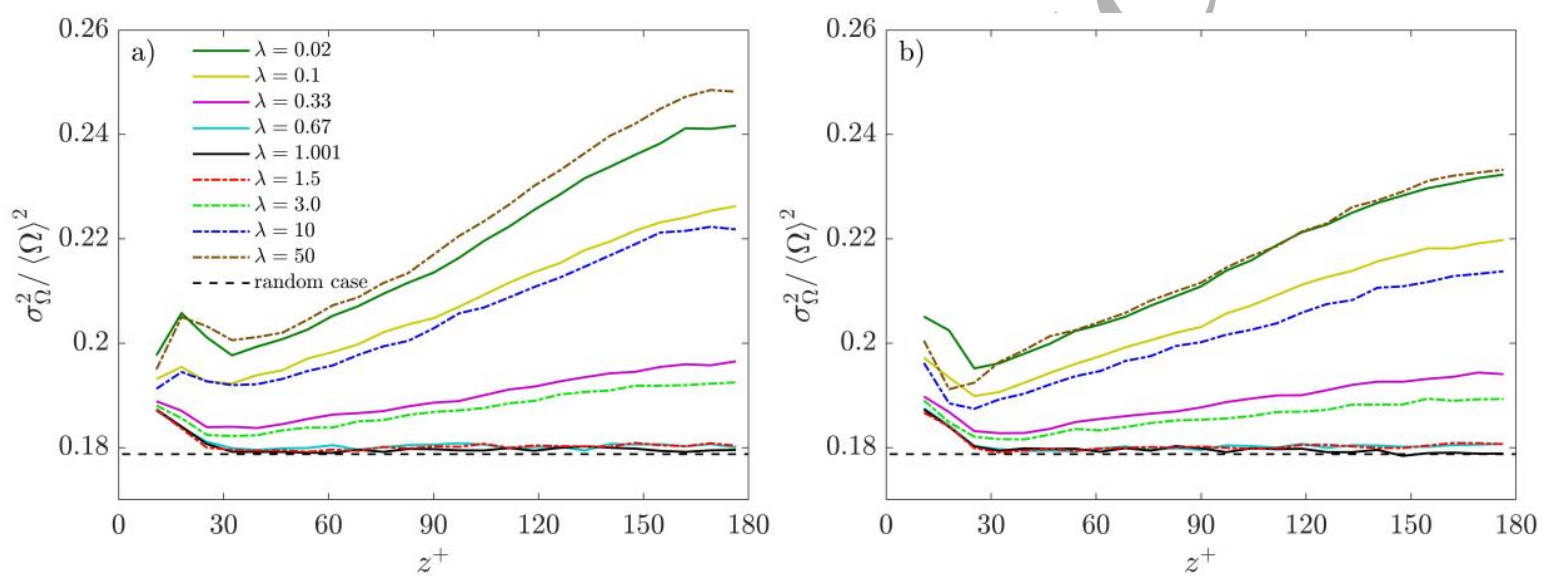

Figure 8. Variance of Voronoi volume $\sigma_{\Omega}{ }^{2}$ normalized by the square of local mean Voronoï volume $\langle\Omega\rangle^{2}$ as a function of the wall-normal coordinate $\mathrm{z}^{+}$for different aspect ratios. (a) Downward flow. (b)

Upward flow. The dashed line represents the case of randomly-distributed particles.

As shown in figure 7 , particles show preferential sampling in both upward and downward flow. The mean velocity difference increases with asphericity, indicating a tendency for spheroidal tracers to sample high-speed regions in downward flow and low-speed regions in upward flow. For all aspect ratios considered in this study, the mean velocity difference reaches a maximum at $z^{+}=15$ and attenuates in the channel center, yet making positive contributions to the sedimentation in both flow configurations.

The non-zero mean velocity difference is closely related to particle clustering. However, it does not precisely provide information about the degree of clustering, because the velocity difference is also affected by the fluctuations of fluid velocity. For instance, a moderate clustering might result in a large velocity difference near the wall, where the fluctuation of velocity is strong. The peak values of the mean velocity difference and streamwise velocity fluctuation are both located at $z^{+} \approx 15$ (Kim et al. 1987), suggesting a relation between these two quantities. To evaluate the degree of clustering based only on the position of the particles, we performed a three-dimensional Voronoï analysis aimed at exploring the clustering that occurs in the homogeneous directions. In particular, we examine the variance of Voronoï volumes, which provides a 
quantitative grid-independent measure of the inhomogeneous distribution of particles within the flow (Monchaux et al. 2010; Nilsen et al. 2013; Tagawa et al. 2012). As shown in figure 8, local clustering is observed for both spheroids and spheres under $z^{+}=30$, where the turbulent structures are most active (Kim et al. 1987). Starting from the channel wall, we find that the variance of Voronoï volumes attenuates as the wallnormal coordinate $\mathrm{z}^{+}$increases, reaching a minimum at about $z^{+}=30$, but then increases again with $z^{+}$, reaching an absolute maximum in the center of channel. Spheres show almost no clustering for $z^{+}>30$, whereas the variance of Voronoï volumes is found to increase with asphericity. These observations are consistent with those reported by Nilsen et al. (2013) for inertial particles, but the variance of Voronoï volumes is smaller in the present cases because tracer particles with modest slip velocity are less prone to cluster. Interestingly, the variance of Voronoï volumes with $z^{+}$indicates that the strongest local clustering occurs in the channel center. In other words, the effect of gravity is more evident in the channel center. We also notice that the variance in downward flow is larger than the one in upward flow in the channel center. This could be explained by two mechanisms. First, the clustering in downward flow is slightly stronger in the center, as indicated by the larger velocity difference shown in figure 7. Second, the value of the variance is known to be proportional to the number density of particles (Nilsen et al. 2013; Romain 2012; Tagawa et al. 2012): In the case of downward flow, particles concentrate in the channel center and result in a higher variance.

\section{Particle transport in the wall-normal direction}

The wall-normal transport of particles, which results in the inhomogeneous distribution in the wallnormal direction, is explained in connection with the slip velocity and local clustering. The mean particle velocity in the wall-normal direction (not shown) remains close to zero because the particle distribution has reached a steady state in the time window used for averaging. However, both the slip velocity and the mean velocity difference have non-zero wall-normal components, which are shown in figure 9 and 10, respectively. According to equation 5 , the slip velocity in the wall-normal direction is determined by particle orientation:

$$
\mathbf{v}_{s, z}=\left[v_{\text {settle }}(0)-v_{\text {settle }}\left(\frac{\pi}{2}\right)\right] \cos \theta_{x} \cos \theta_{z},
$$

where $\theta_{z}$ is the angle between the particle symmetry axis and the wall-normal direction. Non-zero wall-normal slip velocity implies an asymmetric cosine of $\theta_{z}$, which results in particle preferentially drift towards or away from the wall. Moreover, the sign of the wall-normal component of slip velocity (which corresponds to a velocity directed towards the channel center in downward flow and towards the channel walls in upward flow) is consistent with the accumulation. The sign of the mean velocity difference caused by preferential sampling is opposite. Therefore, the accumulation in the wall-normal direction are dominated by slip velocity.

Figure 11 shows the change of particle number density and particle velocity near the wall in upward flow over time. The number density, shown in panel a, keeps increasing until $\mathrm{t}^{+}=900$, which is in agreement with particle accumulation at the wall discussed at the beginning of this section. The process of particle wallnormal transport in upward flow can be described as follows. Once particles are injected randomly in the channel, they begin to drift towards the wall due to the effect of gravity, which is manifested in the negative wall-normal slip velocity shown in figure $11 \mathrm{~b}$. At the same time, particles sample preferentially flow regions in which the fluid velocity is in the opposite direction as the clustering develops. Therefore, a positive mean 
velocity difference is observed, as can be seen in figure $11 \mathrm{c}$. The particle distribution reaches a steady state at $\mathrm{t}^{+} \approx 900$ when the two velocities balance each other. A similar scenario is observed in downward flow (not shown here for sake of brevity) where particles accumulate at the channel center. The transport of particles should be quick since they are inertia-less and move with the turbulent flow with modest slip velocity. Therefore, compared to inertial particles, inertia-less particles are mixed more quickly and reach the steady state earlier.

Particle's preferential sampling of regions along with a specific wall-normal flow direction (to the wall or away from it) is believed to be associated with the sweep and ejection events that characterize wallbounded turbulence, see e.g. Kim et al. (1987). As mentioned above, particles in downward flow sample the high-speed regions and are found more often in wall ward-moving coherent portions of the channel, which correspond to sweep events. Similarly, particles in upward flow sample regions in which ejection events are detected. Yet, such observations do not help us explaining why particles are settling either away or towards the channel walls for opposite bulk flow directions. The effect of turbulent fluctuations on particle motion is complex to analyze. However, a previous study on two-dimensional stationary/channel flow provides a possible explanation (Clifton et al. 2018). In laminar flows with vertical shear, particles rotate periodically and an enhanced settling velocity is observed. Particles have lower angular velocity when they align vertically and sample down-welling regions because of the gravity effect. The mean shear is also vertical in turbulent channel flow, so a similar reasoning is plausible. However, particles no longer rotate periodically in wall turbulence, which provides the possibility of generating clusters. Particles experience shear as they move with the flow, gradually move towards or away from the wall, and finally cluster in down-welling region.
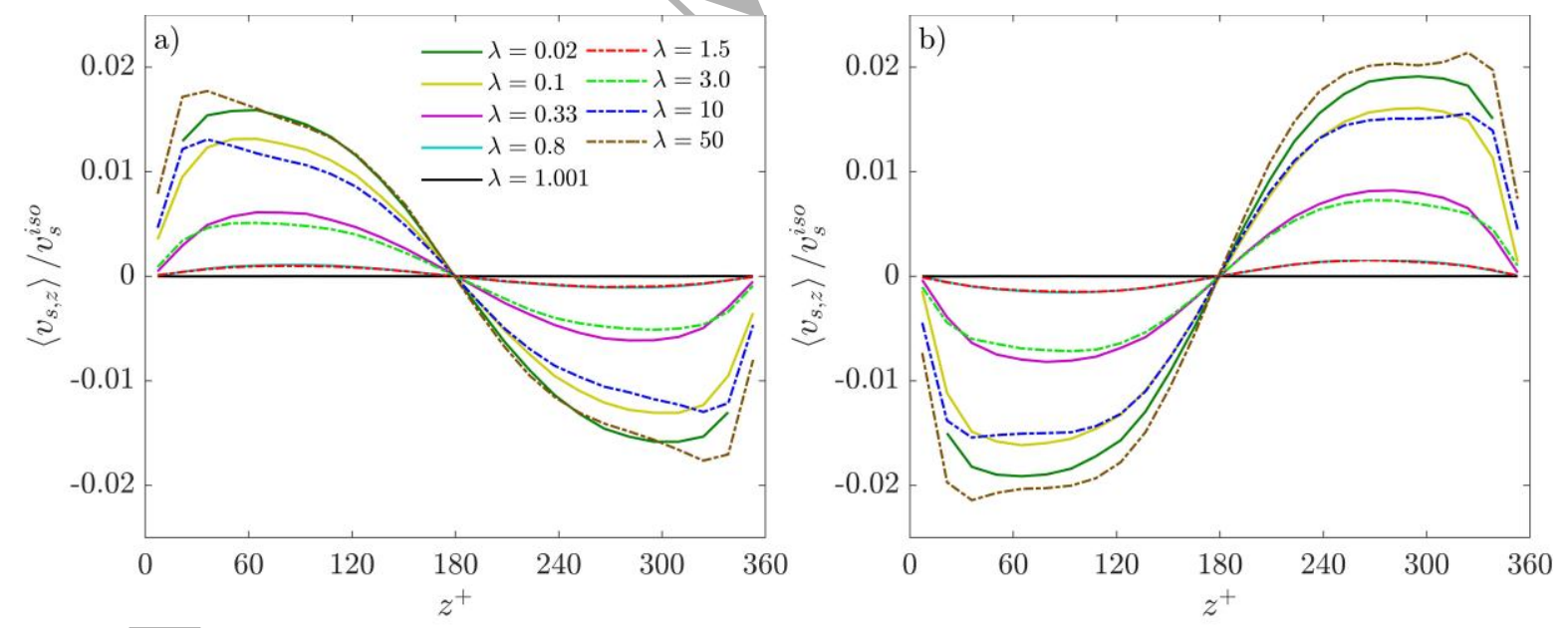

Figure 9. The mean wall-normal slip velocity $\left\langle v_{s, z}\right\rangle$ as function of wall-normal coordinate $\mathrm{z}^{+}$with different aspect ratios. (a) Downward flow. (b) Upward flow. 

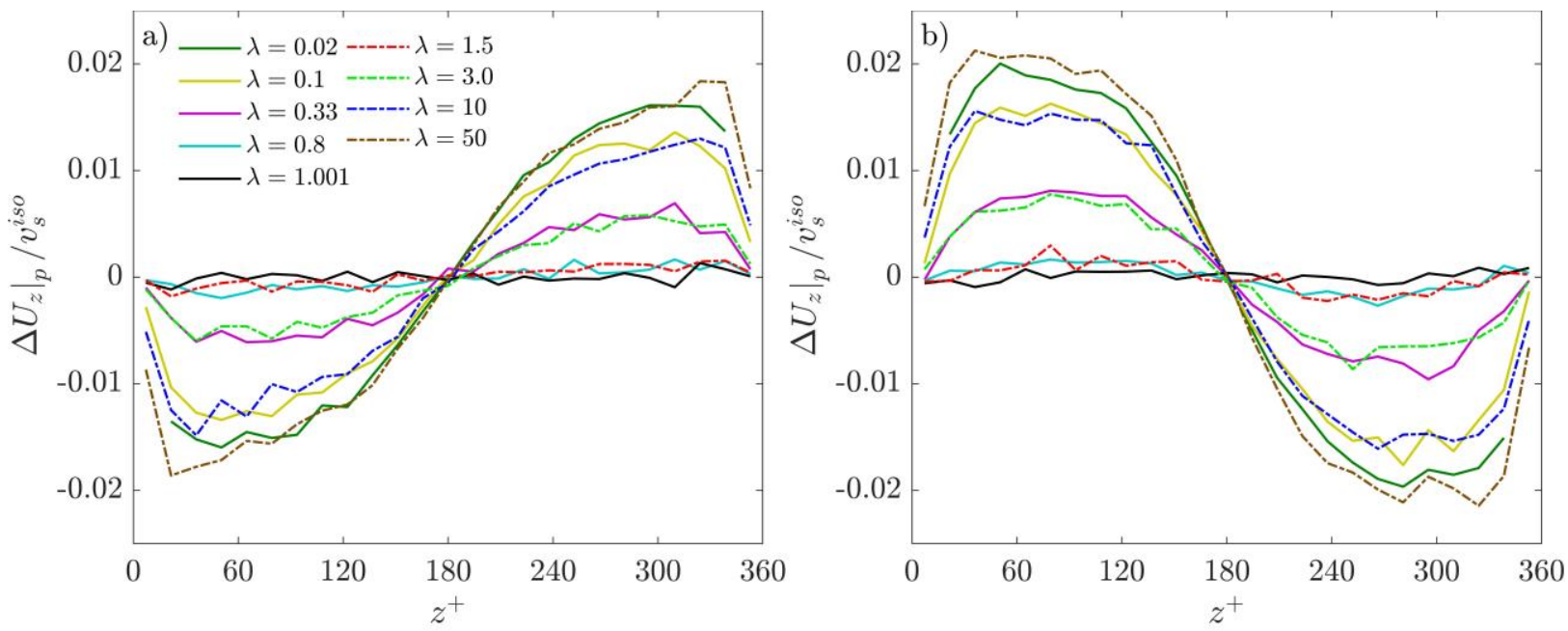

Figure 10. The wall-normal fluid velocity difference $\left.\Delta U_{\mathrm{x}}\right|_{p}$ as function of wall-normal coordinate $\mathrm{z}^{+}$with different aspect ratios. (a) Downward flow. (b) Upward flow.

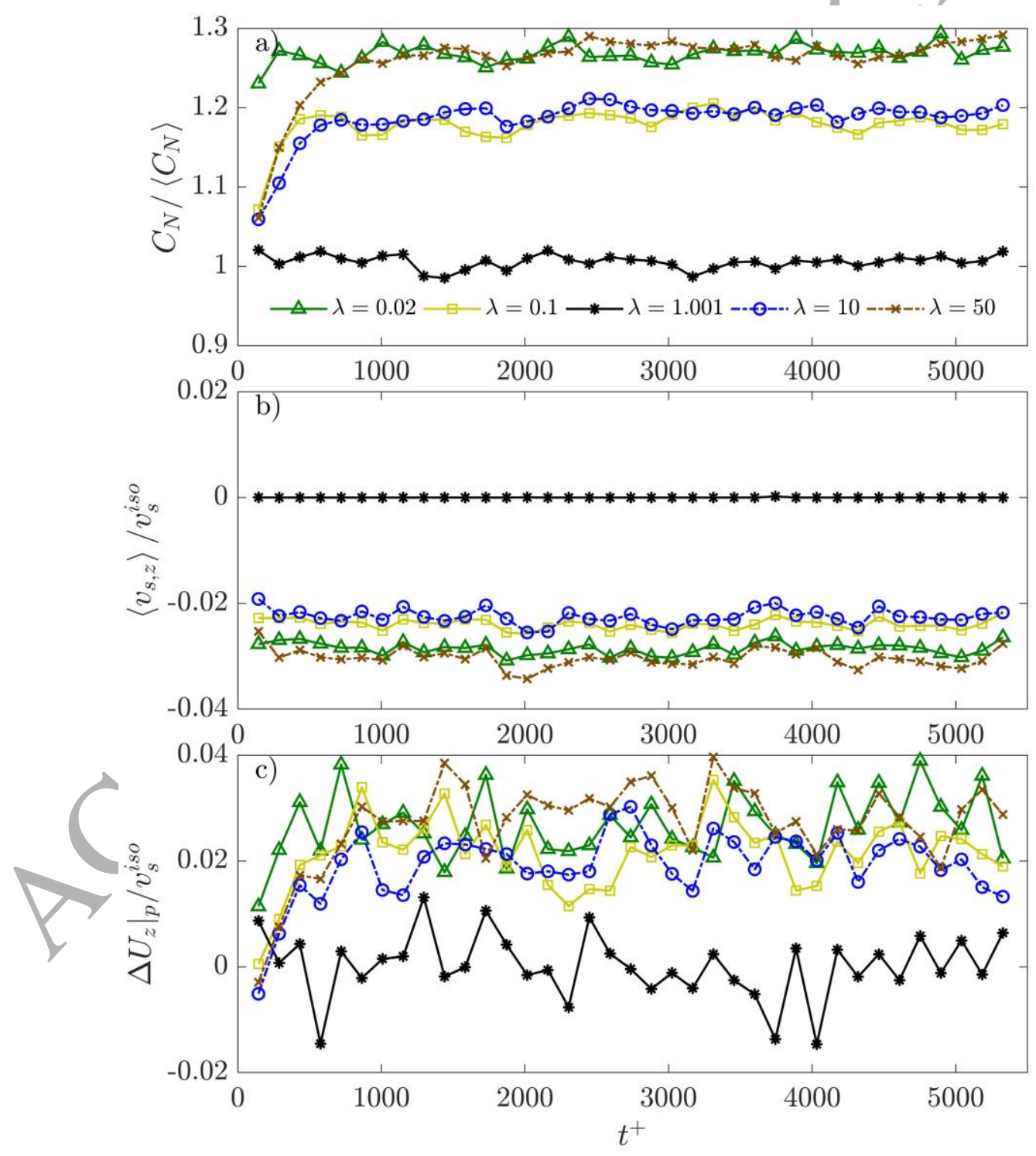

Figure 11. a) Normalized particle number density, b) wall-normal slip velocity and c) wall-normal fluid velocity difference as function of $\mathrm{t}^{+}$in upward flow at $\mathrm{z}^{+}=7.2 \sim 10.8$ (left wall). 


\section{CONCLUDING REMARKS}

We presented direct numerical simulations of settling inertia-less spheroids in vertical channel flow and examined the relationship between settling, clustering and preferential orientation. Particles carried by downward and upward flows are considered, and results compared with the reference no-gravity case. We observe an enhancement of the settling velocity for spheroidal particles, which is explained by two mechanisms. The first one is preferential orientation. As reported in previous studies (Challabotla et al. 2015a; Marchioli et al. 2010), prolate particles tend to align their symmetric axes with the mean flow direction (namely the gravity direction in the present simulations) and oblate particles align perpendicular to that. This preferential orientation results in higher slip velocity along the gravity direction and contributes predominantly to the observed settling velocity enhancement. The second mechanism is local clustering, which originates from particles that, though inertia-less, are able to preferentially sample specific flow regions. This ability comes from the particles' non-zero slip velocity, which allows them to move relative to the surrounding fluid and attain a non-uniform spatial distribution. The results of Voronoï analysis indicate that both spherical and spheroidal particles undergo local clustering near the wall, but only spheroidal particles are observed to cluster in the channel center. Moreover, the mean velocity difference indicates that particles prefer to sample regions of high fluid velocity in downward flow and regions of low fluid velocity in upward flow. Such preferential sampling also contributes to the enhancement of settling. However, the mechanism governing preferential sampling still remains unclear and deserves further investigation.

Another interesting feature of the present physical problem is that particles attain a non-homogeneous distribution (again, not driven by inertia) in the wall-normal direction. Unlike inertia-less particles in gravityfree channel flow, which remain uniformly distributed (Challabotla et. al. 2015), settling particles undergo accumulation in regions of the flow that depend on the flow direction: Particles accumulate in the channel center in downward flow and near the wall in upward flow. This phenomenon also originates from the orientation and local clustering mechanisms. Particles near the wall in upward flow, for instance, have negative mean wall-normal slip velocity because of the preferential orientation and, therefore, drift further towards the wall. When near-wall accumulation increases overtime, local clustering in the regions of higherthan-mean flow velocity also increases. We find that the distribution of particles reaches a steady state when the two mechanisms balance each other. Moreover, our results suggest that local clustering is related to the sweep and ejection events characterizing near-wall turbulence. For instance, particles in upward flow sample down-welling regions, namely low-speed regions. These regions are related to the occurrence of ejection events and, therefore, particles sample regions where the wall-normal fluid velocity is directed towards the channel center. The same analysis is applicable to the cases of particles in downward flow.

In spite of the findings discussed in this paper, some questions still remain unanswered. In particular, how does the non-zero slip velocity affect the motion of each particle, and further the macroscopic preferential orientation that we observe? How to explain the dependence of preferential sampling on the flow direction? These issues need to be further studied and might be important for improving our understanding of settling particles with zero or negligible inertia. 


\section{ACKNOWLEDGEMENT}

This work is supported by the National Natural Science Foundation of China under grant Nos. 11702158, 91752205, and 11490551 and from Microsabbatical of NTNU Digital. HIA is grateful to the support by the Research Council of Norway through research grant no. 250744. The Research Council of Norway (Programme for Supercomputing, grant No. NN2649K) are also greatly acknowledged.

\section{References:}

Andrady, A.L., 2011. Microplastics in the marine environment. Mar. Pollut. Bull. 62, 1596-1605.

Ardekani, M.N., Sardina, G., Brandt, L., Karp-Boss, L., Bearon, R.N., Variano, E.A., 2017. Sedimentation of inertia-less prolate spheroids in homogenous isotropic turbulence with application to non-motile phytoplankton. J. Fluid Mech. 831, 655-674.

Butler, J.E., Shaqfeh, E.S.G., 2002. Dynamic simulations of the inhomogeneous sedimentation of rigid fibres. J. Fluid Mech. 468, 205-237.

Challabotla, N.R., Zhao, L., Andersson, H.I., 2015b. Orientation and rotation of inertial disk particles in wall turbulence. J. Fluid Mech. 766, R2.

Challabotla, N.R., Zhao, L., Andersson, H.I., 2016. On fiber behavior in turbulent vertical channel flow. Chem. Eng. Sci. 153, 75-86.

Chen, C., Yeh, K., Aisyah, R., Lee, D., Chang, J., 2011. Cultivation, photobioreactor design and harvesting of microalgae for biodiesel production: A critical review. Bioresource Technol. 102, 71-81.

Clifton, W., Bearon, R.N., Bees, M.A., 2018. Enhanced sedimentation of elongated plankton in simple flows. Ima J. Appl. Math. 83, 743-766.

Cole, M., Lindeque, P., Fileman, E., Halsband, C., Goodhead, R., et al., 2013. Microplastic ingestion by zooplankton. Environ. Sci. Technol. 47, 6646-6655.

Cui, Y., Ravnik, J., Hriberšek, M., Steinmann, P., 2018. A novel model for the lift force acting on a prolate spheroidal particle in an arbitrary non-uniform flow. Part I. Lift force due to the streamwise flow shear. Int. J. Multiphas. Flow 104, 103-112.

Ducklow, H.W., Purdie, D.A., Williams, P.J.L., Davies, J.M., 1986. Bacterioplankton: A Sink for carbon in a coastal marine plankton community. Science $232,865$.

Fornari, W., Ardekani, M.N., Brandt, L., 2018. Clustering and increased settling speed of oblate particles at finite Reynolds number. J. Fluid Mech. 848, 696-721.

Fornari, W., Picano, F., Brandt, L., 2016. Sedimentation of finite-size spheres in quiescent and turbulent environments. J. Fluid Mech. 788, 640-669.

Guazzelli, É., 2006. Sedimentation of small particles: how can such a simple problem be so difficult? Comptes Rendus Mécanique 334, 539-544.

Gustavsson, K., Tornberg, A.K., 2009. Gravity induced sedimentation of slender fibers. Phys. Fluids 21, 123301.

Harding, G.C.H., 1974. The food of deep-sea copepods. J. Mar. Biol. Assoc. Uk 54, 141-155.

Herzhaft, B., Guazzelli, E., 1999. Experimental study of the sedimentation of dilute and semi-dilute suspensions of fibres. J. Fluid Mech. 384, 133-158.

Herzhaft, B., Guazzelli, É., Mackaplow, M.B., Shaqfeh, E.S.G., 1996. Experimental Investigation of the Sedimentation of a Dilute Fiber Suspension. Phys. Rev. Lett. 77, 290-293.

Kim, J., Moin, P., Moser, R., 1987. Turbulence statistics in fully developed channel flow at low Reynolds number. J. Fluid Mech. 177, 133-166.

Mackaplow, M.B., Shaqfeh, E.S.G., 1998. A numerical study of the sedimentation of fibre suspensions. J. Fluid Mech. 376, 149-182.

Marchioli, C., Zhao, L., Andersson, H.I., 2016. On the relative rotational motion between rigid fibers and fluid in turbulent channel flow. Phys. Fluids 28, 013301.

Monchaux, R., Bourgoin, M., Cartellier, A., 2010. Preferential concentration of heavy particles: A Voronoï analysis. Phys. Fluids 22, 103304.

Nilsen, C., Andersson, H.I., Zhao, L., 2013. A Voronoï analysis of preferential concentration in a vertical channel flow. Phys. Fluids 25, 115108.

Romain, M., 2012. Measuring concentration with Voronoï diagrams: the study of possible biases. New J. Phys. 14, 095013.

Shanks, A.L., Trent, J.D., 1980. Marine snow: sinking rates and potential role in vertical flux. Deep Sea Research Part A. Oceanographic Research Papers 27, 137-143.

Shapiro, M., Goldenberg, M., 1993. Deposition of glass fiber particles from turbulent air flow in a pipe. J. Aerosol Sci. $24,65-87$. 
Siewert, C., Kunnen, R.P.J., Meinke, M., Schröder, W., 2014. Orientation statistics and settling velocity of ellipsoids in decaying turbulence. Atmos. Res. 142, 45-56.

Smith, M., Love, D.C., Rochman, C.M., Neff, R.A., 2018. Microplastics in seafood and the implications for human health. Current Environmental Health Reports 5, 375-386.

Tagawa, Y., Mercado, J.M., Prakash, V.N., Calzavarini, E., Sun, C., Lohse, D., 2012. Three-dimensional Lagrangian Voronoï analysis for clustering of particles and bubbles in turbulence. J. Fluid Mech. 693, 201-215.

Uduman, N., Qi, Y., Danquah, M.K., Forde, G.M., Hoadley, A., 2010. Dewatering of microalgal cultures: A major bottleneck to algae-based fuels. J. Renew Sustain Ener 2, 012701.

Van Cauwenberghe, L., Vanreusel, A., Mees, J., Janssen, C.R., 2013. Microplastic pollution in deep-sea sediments. Environ. Pollut. 182, 495-499.

Voth, G.A., Soldati, A., 2017. Anisotropic Particles in Turbulence. Annu Rev Fluid Mech 49, 249-276.

Wang, L., Maxey, M.R., 1993. Settling velocity and concentration distribution of heavy particles in homogeneous isotropic turbulence. J. Fluid Mech. 256, 27-68.

Yuan, W., Andersson, H.I., Zhao, L., Challabotla, N.R., Deng, J., 2017. Dynamics of disk-like particles in turbulent vertical channel flow. Int. J. Multiphas. Flow 96, 86-100.

Zhao, L., Andersson, H.I., 2016. Why spheroids orient preferentially in near-wall turbulence. J. Fluid Mech. 807, 221234. 